MPP-2009-160

\title{
NLO electroweak contributions to squark pair production at the LHC
}

\author{
Jan Germer, Wolfgang Hollik, Edoardo Mirabella and Maike K. Trenkel \\ Max-Planck-Institut für Physik, Föhringer Ring 6, D-80805 München, Germany
}

\begin{abstract}
We present the tree-level and next-to-leading order (NLO) electroweak (EW) contributions to squark-squark production at the Large Hadron Collider (LHC) within the framework of the Minimal Supersymmetric Standard Model (MSSM).
\end{abstract}

Keywords: MSSM, NLO Computations, Hadronic Colliders

PACS: $12.15 . \mathrm{Lk}$

\section{INTRODUCTION}

If supersymmetry is realized at the TeV scale it will be probed at the LHC. The direct search for colored SUSY particles, i.e. squarks and gluinos, is of special interest owing to their large production rate at hadron colliders.

As known from stop-anti-stop [1], squark-anti-squark [2] and squark-gluino [3] production, NLO electroweak (EW) contributions of $\mathscr{O}\left(\alpha_{s}^{2} \alpha\right)$ were found to be significant in specific configurations, and of comparable size as the tree-level EW contributions of $\mathscr{O}\left(\alpha_{S} \alpha+\alpha^{2}\right)$ [4]. EW effects to gluino-gluino production are only present at the loop level, and were found to be small [5].

Here we focus on the process of squark-squark production:

$$
\mathrm{PP} \rightarrow \tilde{\mathrm{Q}}_{\alpha} \tilde{\mathrm{Q}}_{\beta}^{\prime}+X, \quad\left(\tilde{\mathrm{Q}}^{(\prime)} \neq \tilde{\mathrm{t}}, \tilde{\mathrm{b}}\right),
$$

where $\{\alpha, \beta\}=\{\mathrm{L}, \mathrm{R}\}$ label the chirality of the squarks, neglecting left-right mixing. The final state squarks have to be of the same generation as the initial state quarks. We do not consider the production of top (bottom) squarks due to the vanishing (small) density of the corresponding quark inside the proton. In total one has to consider 36 distinct processes, resulting from the various possible combinations of squarks of different flavor and chirality in the final state.

In the following we will consider the tree-level and next-to-leading order electroweak contributions to the processes (1). This work is a yet missing part of our ongoing project on the computation of the complete EW contributions up to $\mathscr{O}\left(\alpha_{s}^{2} \alpha\right)$ to all squark and gluino pair production processes at the LHC. 


\section{TREE-LEVEL EW CONTRIBUTIONS}

In general, squark-squark production processes can be divided into three classes according to the flavor of the produced squarks:

1. Production of two same-flavor squarks, e.g. $\mathrm{PP} \rightarrow \tilde{\mathrm{u}}_{\alpha} \tilde{\mathrm{u}}_{\beta}, \tilde{\mathrm{d}}_{\alpha} \tilde{\mathrm{d}}_{\beta}, \ldots$

2. Production of two squarks of different flavor, belonging to the same $\mathrm{SU}(2)$ doublet, $\mathrm{PP} \rightarrow \tilde{\mathrm{u}}_{\alpha} \tilde{\mathrm{d}}_{\beta}, \tilde{\mathrm{c}}_{\alpha} \tilde{\mathrm{s}}_{\beta}$.

3. Production of different squarks in different $S U(2)$ doublets, e.g. $\mathrm{PP} \rightarrow \tilde{\mathrm{u}}_{\alpha} \tilde{\mathrm{c}}_{\beta}, \tilde{\mathrm{u}}_{\alpha} \tilde{\mathrm{s}}_{\beta}, \ldots$

At tree-level, squark-squark production can only be induced via gluino, neutralino or chargino exchange in the $\mathrm{t}$ - or u-channel. In the following we will refer to a diagram with gluino (neutralino/chargino) exchange as a QCD diagram (EW diagram). The leading order cross section of $\mathscr{O}\left(\alpha_{s}^{2}\right)$ as well as a contribution of $\mathscr{O}\left(\alpha^{2}\right)$ is present in all three classes through the squared QCD and EW matrix elements, respectively. In the first two classes, one also finds a contribution of $\mathscr{O}\left(\alpha_{s} \alpha\right)$ from the non-vanishing interference of QCD and EW diagrams.

\section{NLO EW CONTRIBUTIONS}

The NLO EW contributions of $\mathscr{O}\left(\alpha_{s}^{2} \alpha\right)$ consist of virtual corrections and of real corrections from photon, gluon, and quark emission. Various interference terms have to be selected carefully to get an IR finite result in the aimed order in perturbation theory.

\section{Virtual corrections}

Three different types of interference terms constitute the virtual corrections at $\mathscr{O}\left(\alpha_{s}^{2} \alpha\right)$ :

- The interference contributions of tree-level QCD diagrams with one-loop graphs obtained from tree-level QCD diagrams with EW insertions.

- The interference contributions of tree-level QCD diagrams with one-loop graphs obtained from tree-level EW diagrams with QCD insertions.

- The interference contributions of tree-level EW diagrams with one-loop graphs obtained from tree-level QCD diagrams with QCD insertions.

The on-shell scheme is used to renormalize the masses and the wave functions of the quarks, squarks and gluinos $[6,7]$. The strong coupling constant is renormalized in the $\overline{\mathrm{MS}}$ scheme with five flavor running of $\alpha_{s}$. SUSY Slavnov-Taylor identities are restored by adding a proper counterterm for the squark-quark-gluino coupling $[8,9]$. 
TABLE 1. Different chiral contributions to the total hadronic cross section for squark-squark production at the LHC. The result is implicitly summed over all possible flavors $\tilde{\mathrm{q}}, \tilde{\mathrm{q}}^{\prime}$. $\delta_{\text {tree }}=\sigma_{\alpha_{s} \alpha+\alpha^{2}}^{\text {Tree EW }} / \sigma_{\alpha_{s}^{2}}^{\text {Born }}$ and $\delta_{\text {tree }+\mathrm{NLO}}=\sigma_{\alpha_{s}^{2} \alpha}^{\mathrm{NLO} \text { EW }} / \sigma_{\alpha_{s}^{2}}^{\text {Born }}$.

\begin{tabular}{c|r|r|r|r|r} 
& $\sigma_{\alpha_{s}^{2}}^{\text {Born }}$ & $\sigma_{\alpha_{s} \alpha+\alpha^{2}}^{\text {Tree EW }}$ & $\sigma_{\alpha_{s}^{2} \alpha}^{\mathrm{NLO}}$ & $\delta_{\text {tree }}$ & $\delta_{\text {tree }+ \text { NLO }}$ \\
\hline$\tilde{\mathrm{q}}_{\mathrm{L}} \tilde{\mathrm{q}}_{\mathrm{L}}^{\prime}$ & $1632 . \mathrm{fb}$ & $364 . \mathrm{fb}$ & $-71 . \mathrm{fb}$ & $22.3 \%$ & $18.0 \%$ \\
\hline$\tilde{\mathrm{q}}_{\mathrm{L}} \tilde{\mathrm{q}}_{\mathrm{R}}^{\prime}$ & $1682 . \mathrm{fb}$ & $2 . \mathrm{fb}$ & $-69 . \mathrm{fb}$ & $0.1 \%$ & $-3.9 \%$ \\
\hline$\tilde{\mathrm{q}}_{\mathrm{R}} \tilde{\mathrm{q}}_{\mathrm{R}}^{\prime}$ & $1876 . \mathrm{fb}$ & $31 . \mathrm{fb}$ & $2 . \mathrm{fb}$ & $1.7 \%$ & $1.6 \%$ \\
\hline$\tilde{\mathrm{q}} \tilde{\mathrm{q}}^{\prime}$ & $5189 . \mathrm{fb}$ & $397 . \mathrm{fb}$ & $-141 . \mathrm{fb}$ & $7.7 \%$ & $4.9 \%$
\end{tabular}

\section{Real corrections}

To obtain IR and collinear finite results, the processes of real photon and real gluon emission have to be included. At $\mathscr{O}\left(\alpha_{s}^{2} \alpha\right)$, the former is given by the squared matrix element of tree-level QCD diagrams radiating a photon, while the latter is obtained by the interference contributions of EW and QCD tree-level diagrams radiating a gluon. IR and collinear singularities have been regularized using mass regularization. The EW contribution (QCD contribution), which is given by the sum of real photon emission (real gluon emission) and EW (QCD) loop insertions, is IR safe. The remaining universal collinear singularities are absorbed into the definition of the quark distribution functions (PDF). We also consider real quark emission at $\mathscr{O}\left(\alpha_{s}^{2} \alpha\right)$. At this order, only universal collinear singularities arise that also have to be absorbed by redefining the quark PDF. In specific scenarios, internal gluinos, neutralinos or charginos can go on shell if they are heavier than one of the produced squarks. If this is the case, we regularize the singularity by including the respective particle width in the resonant propagator.

\section{NUMERICAL RESULTS}

Diagrams and corresponding amplitudes were generated using FeynArts $[10,11]$ while the algebraic simplifications and numerical evaluation of the scalar integrals were performed using FormCalc and LoopTools $[12,13]$. For illustration of the EW effects, we consider the SPS1a' point of the MSSM, suggested by the SPA convention [14].

In Table 1 we give results for the integrated total cross section. We refer to the three different combinations of chiralities of the produced squarks (LL, LR, RR) and to the inclusive $\tilde{\mathrm{q}} \tilde{\mathrm{q}}^{\prime}$ process, respectively. The tree-level EW contributions are positive and mainly given by the production of two left-handed (LH) squarks with a small contribution given by the production of two right-handed $(\mathrm{RH})$ squarks. The NLO EW corrections are negative and equally constituted by the corrections to the production of two LH squarks and two squarks of different chiralities. In contrast, the tree-level EW contribution to the production of squarks of different chiralities and the NLO EW contribution for two RH squarks is negligible. This reflects the non-trivial behavior of the NLO EW corrections; it is not possible to give a general correction factor to the tree-level EW results. The impact of the EW contributions on the inclusive total cross 
FIGURE 1. Distribution of the average $\mathrm{p}_{\mathrm{T}}$ of the squark for $\tilde{u}_{\mathrm{L}} \tilde{u}_{\mathrm{L}}$ production at the LHC.

(a)

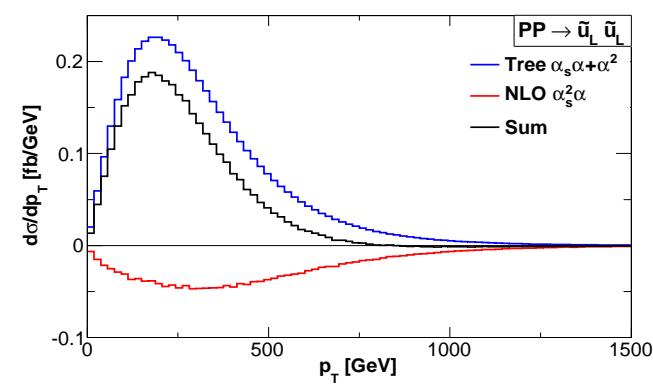

(a) The tree-level EW and the NLO EW contribution, as well as the sum of both.

(b) Different contributions of $\mathscr{O}\left(\alpha_{s}^{2} \alpha\right)$.

(c) Relative contribution of the NLO EW corrections, $\delta=\mathscr{O}\left(\alpha_{s}^{2} \alpha\right) / \mathscr{O}\left(\alpha_{s}^{2}\right)$. (b)

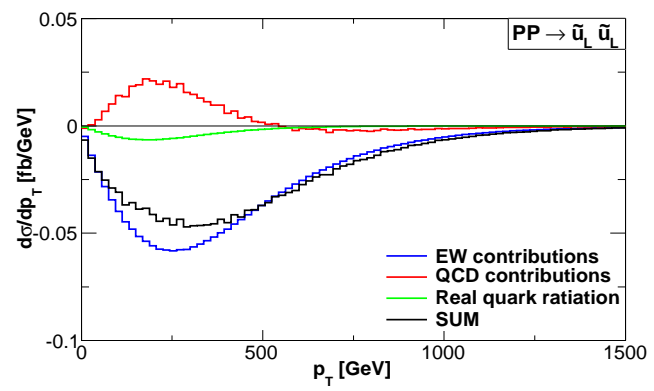

(c)

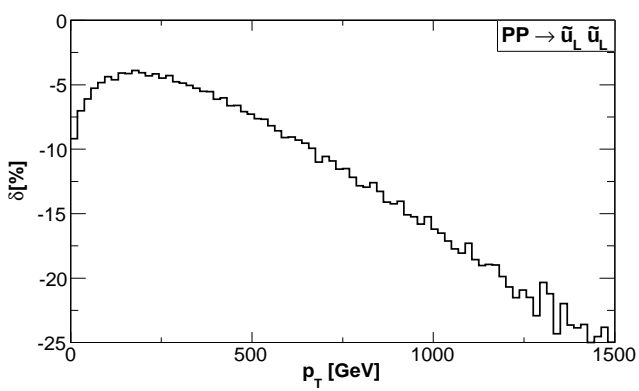

section reduces from $7.7 \%$ to $4.9 \%$ if NLO EW corrections are included. To illustrate the significance of EW contributions on differential distributions we show in Figure 1 the differential $\mathrm{p}_{\mathrm{T}}$ distribution for $\tilde{\mathrm{u}}_{\mathrm{L}} \tilde{\mathrm{u}}_{\mathrm{L}}$ production. At low $\mathrm{p}_{\mathrm{T}}$, the EW and QCD contributions have different sign, and therefore partially cancel in the sum. As usual, as one can see in Figure 1c, NLO EW corrections become more important in the high $\mathrm{p}_{\mathrm{T}}$ region.

\section{REFERENCES}

1. W. Hollik, M. Kollar, and M. K. Trenkel, JHEP 02, 018 (2008), 0712.0287.

2. W. Hollik, and E. Mirabella, JHEP 12, 087 (2008), 0806.1433.

3. W. Hollik, E. Mirabella, and M. K. Trenkel, JHEP 02, 002 (2009), 0810.1044.

4. S. Bornhauser, M. Drees, H. K. Dreiner, and J. S. Kim, Phys. Rev. D76, 095020 (2007), 0709.2544.

5. E. Mirabella (2009), 0908.3318.

6. A. Denner, Fortschr. Phys. 41, 307-420 (1993), 0709.1075.

7. W. Hollik, and H. Rzehak, Eur. Phys. J. C32, 127-133 (2003), hep-ph/ 0305328.

8. W. Beenakker, R. Hopker, M. Spira, and P. M. Zerwas, Nucl. Phys. B492, 51-103 (1997), hep-ph / 9610490.

9. W. Hollik, and D. Stockinger, Eur. Phys. J. C20, 105-119 (2001), hep-ph/0103009.

10. T. Hahn, Comput. Phys. Commun. 140, 418-431 (2001), hep-ph/ 0012260.

11. T. Hahn, and C. Schappacher, Comput. Phys. Commun. 143, 54-68 (2002), hep-ph/ 0105349.

12. T. Hahn, and M. Perez-Victoria, Comput. Phys. Commun. 118, 153-165 (1999), hep-ph/ 9807565.

13. T. Hahn, and M. Rauch, Nucl. Phys. Proc. Suppl. 157, 236-240 (2006), hep-ph / 0601248.

14. B. C. Allanach, et al., Eur. Phys. J. C25, 113-123 (2002), hep-ph/ 0202233. 